\title{
The Cost-Benefit Analysis for Bananas Diversity Production in China Foc. Zones
}

\author{
Linbing Xu, Bingzhi Huang*, Yuanli Wu, Yonghong Huang, Tao Dong \\ Banana Research Center, Fruit Research Institute, Guangdong Academy of Agricultural Sciences, Guangzhou, China. \\ Email: ${ }^{*} x u l i n b i n g @ 163 . n e t$
}

Received December $24^{\text {th }}, 2010$; revised March $10^{\text {th }}, 2011$; accepted July $11^{\text {th }}, 2011$.

\begin{abstract}
Over 50 years ago, banana export plantations in Panama were ruined by Fusarium wilt race 1 (Foc. R1) since the popular cultivar Gros Michel is susceptible to Foc. R1 [1]. Fortunately, the resistant cultivar Cavendish replaced Gros Michel as the world biggest commercial fruit after ten years' research and development. Due to its good economic profit, banana industry develops very fast recently in China. Unfortunately, Fusarium oxysporum f. sp. cubense tropical race 4 (TR4) brought disaster to Cavendish plantation since 1996. Most of traditional banana planting zones of Guangdong and Hainan provinces were infected by Foc. TR4 to the end of 2009. In order to fight against Foc. TR4, here we tried to investigate the cost-benefit and disease resistance of six banana cultivars (Cavendish Baxijiao, Dajiao, Guangfen No. 1, Fenzha No. 1, Gongjiao, Haigongjiao) planted in China Foc. Zones. Comparisons were conducted on their economic characteristics, cost, benefit, as well as the advantage and disadvantage and their application in rotation. Generally, supposing the investment of \$ 4400/ha, the banana plantation attains a good economic profit as \$ 2200/ha. Rotation of these cultivars can maintain the biodiversity as well as improve the sustainable development of banana industry.
\end{abstract}

Keywords: Banana, Diversity, Foc. TR 4 Zone, Cost-Benefit, Disease Resistance

\section{Introduction}

Banana is the biggest tropical fruit in China, which covers an area of 350,224 ha, production of 9,006,450 tones fruit in 2009 [2], promoting employment of over 2 million people. The good economic profit of banana cultivation has stimulated the rapid development of banana production in recent years. However, since 1996, the outbreak of Fusarium wilt (Fusarium oxysporum f. sp. cubense "tropical" race 4) (Foc. TR4) spread quickly in the main production areas of Cavendish [3]. According to incomplete statistics, an areas of 200,000 hectares in Guangdong and Hainan provinces have been affected. The land suitable for banana planting was gradually reduced and the banana industry is facing the fate as Panama's banana industry did 50 years ago [1]. More than 30 varieties were evaluated for resistance to Foc. in Guangdong province, south China [4]. In this paper, commercial banana plantations with an area of 10 ha were investigated in Guangdong and Hainan during 2008-2009. Economic characteristics, production costs and efficiency of cultivars such as: Baxijiao (AAA Cavendish), Dajiao (ABB P. Kapuk), Guangfen No. 1
(ABB P. Awak), Fenzha No. 1 (ABB P. Awak), Gongjiao (AA P. Mas), Haigongjiao (AA P. Lampung) were analysed. We compared their advantages in production, suitable planting areas as well as marketing potential. The existing problems for further development of banana production is discussed. The commodity value chains were proposed to promote the sustainable development of banana industry.

\section{Economic Characteristics of Banana Cultivars in China}

Biological characteristics and economic characteristics of banana cultivars are very important for farmers. The investigation conducted in the commercial plantations in Guangdong and Hainan provinces focus on the items as planting density, bunch mass, harvest rate, yield, life cycle, ratoon cycle, susceptibility of Foc. Race 1 (R1) \& TR4. Comparisons are shown in Table 1.

\subsection{Cultivars and the Evaluation}

Baxijiao is the most popular banana cultivar in China which account for $50 \%$ the area under banana, with 
Table 1. Economic characteristics of main banana cultivars in China.

\begin{tabular}{|c|c|c|c|c|c|c|c|c|}
\hline Cultivars & $\begin{array}{c}\text { Density } \\
\text { (plants/ha) }\end{array}$ & $\begin{array}{l}\text { Bunchmass } \\
\quad(\mathrm{kg})\end{array}$ & $\begin{array}{l}\text { Harvest } \\
\text { Rate }(\%)\end{array}$ & Yield (kg/ha) & $\begin{array}{l}\text { Life cycle } \\
\text { (d) }\end{array}$ & $\begin{array}{c}\text { Ratoon harvest } \\
\text { Rate }(\%)\end{array}$ & $\begin{array}{l}\text { Ratoon cycle } \\
\text { (d) }\end{array}$ & Foc. susceptible \\
\hline Cavendish Baxijiao & 2010 & 21.8 & 92.5 & 40531.5 & 316 & 85.9 & 235 & $\begin{array}{c}\text { Resistant to R1 high suscept. } \\
\text { TR4 }\end{array}$ \\
\hline Dajiao & 1590 & 17.5 & 98.5 & 27408 & 405 & 97 & 275 & Hi. resistant \\
\hline Guangfen No. 1 & 1650 & 26.0 & 71.7 & 30759 . & 472.5 & - & - & Hi. suscept. R1 \& TR4 \\
\hline Fenzha No. 1 & 2000 & 13.2 & 96.8 & 25551 & 403 & 100 & 263 & $\begin{array}{l}\text { Hi. resistant to TR4, resistant to } \\
\text { R1 }\end{array}$ \\
\hline Gongjiao & 2475 & 11.0 & 91 & 24766.5 & 303 & 85 & 233 & $\begin{array}{l}\text { Resistant to R1 Suscept. to TR } \\
4\end{array}$ \\
\hline Haigongjiao & 3030 & 7.67 & 96.7 & 22473 & 257 & 100 & 165 & Resistant to R1 \& TR4 \\
\hline
\end{tabular}

*The life cycle means the average time from plantlet planting to harvest. The ratoon cycle means the average time from first crop harvested to ratoon harvested.

higher-yield, better quality, larger market and stable purchase price. However, it is very susceptible to Foc. TR4. Dajiao is widely adapted tolerant, disease resistant and wind resistance. But it tastes sour, and is only sold in Guangdong and the Hong Kong market with low price. Guangfen No. 1 was screened by Huang Bingzhi, Scientist of Guangdong Academy of Agricultural Sciences in the 1990s. It is variety with high-yield and better quality. But it is also susceptible to fursarium wilt. Fenzha No. 1 (MUSA ABB Fenjiao) (Guangfen No. 2) came from hybridization of $q$ MUSA ABB Fenjiao X $\curvearrowright$ MUSA BBw Balbisiana, crossed by Li Fengnian, scientist of Guangdong Academy of Agricultural Sciences in the 1990s. The hybrid is resistant to fungus diseases (Foc. Sigatoka, Freckle, BBTV, CMV. etc.), with good quality fruit and shorter life cycle compare to Guangfen No. 1. But the yield is low. Gongjiao keeps the best quality and highest price of bananas, but is low-yielding and susceptible to Foc. TR4. Haigongjiao was introduced from Southeast Asia, fruit cracking in autumn and winter, with a shorter life cycle. The shelf life. It is resistant to Foc. TR4 [5].

\subsection{Planting Density, Bunch Mass, Yield, Plant Crop and Ratoon Harvest Rate}

Planting density was investigated on the Pearl River Delta and Hainan Island. The planting density of Baxijiao is ranged from 1650 - 2550/ha. Dajiao is only planted commercially in Pearl River Delta; Guangfen No. 1 's planting density is 1500 - 1800/ha. Fenzha No. 1's planting density was 1800 - 2175/ha. Gongjiao's planting density was 2100 - 3450/ha. Haigongjiao's planting density range $2700-3600 /$ ha. Ratoon suckers were retained mainly as the mother plant's density. About 10\% - 40\% twin suckers were retained to replace Foc. and virus diseases infected (BBTV, CMV) damaged plants.

Bunch mass is related to the cultivar and season, less during December to May, more during June to November. Ratoon would weight about 30\% - 50\% more than plant crop. The bunch mass in Table $\mathbf{1}$ is the average of plant crop and ratoon. Among Dajiao, Dongguan Dajiao yielding more than Panyu Dajiao. Guangfen No.1 is which the best one among Fengjiaos. Which about $10 \%$ more than other Fenjiao. Fengzha No. 1 yield of $10 \mathrm{~kg} / \mathrm{bunch}$ during winter-spring, and $20 \mathrm{~kg} / \mathrm{bunch}$ in summer-autumn. Gongjiao yield is $5-16 \mathrm{~kg} /$ bunch. Haigongjiao yield is 4 - $14 \mathrm{~kg} /$ bunch.

The harvest rates of the plant crop and the ratoon are related to the former crop and soil type. If a few plants are infected by Foc. TR4 in Baxijiao and Gongjiao plantation, they may not remain an ratoon crop. Incase the incidences of Foc. is more than $10 \%$ that the plantation can not be ratooned, since the ratoon harvest rates may be as low as $40 \%$. Guangfen No. 1 can only be planted in the land free of cultivation of Cavendish and Fenjiao, when the incidences of Foc. are more than $10 \%$ of the Fenjiao plantation that can not be ratooned. Fenzha No. 1 and Haigongjiao can be ratooned.

The mortality of Foc. TR4, CMV, BBTV in Baxijiao normally is bout $3 \%-60 \%$. Twin suckers can increase the number of harvested bunches. Foc. TR4 spread in basalt laterite soil are slower than in sa Guangfen ndy loam. Ratoons depend on soil types. Dajiao generally can be harvested $100 \%$; No. 1 can be generally harvested around $60 \%$ if there is no special disinfection and prevention of treatment. Fenzha No. 1 and Haigongjiao on the 1 st harvest rates can generally be more than $95 \%$, through the appropriate remaining twin suckers can be harvested more than $100 \%$. Gongjiao is more resistant to Foc. TR4 than Cavendish, particular in Laterite.

Yield is the number of plants multiplied by bunch mass, and the harvest rate. The production of Guangfen No. 1, Braxijiao, Gongjiao may decrease 10\% - 30\% because of harvest rate. While Guangfen No. 1 is harvested only one crop, Baxijiao, Gongjiao can be harvest two crops. Fenzha No. 1, Haigongjiao can be ratooned. 


\subsection{Life Cycle and Ratoon Cycle}

The life cycle and ratoon cycle of Braxijiao are 316 days and 235 days respectively. Dajiao is 405 days and 275 days, respectively. Guangfen No. 1 is 472.5 days. Fenzha No. 1 is 403 days and 263 days respectively. Gongjiao are 303 days and 233 days respectively. Haigongjiao are 257 days and 165 days respectively.

\subsection{The Susceptibility of Foc.}

The susceptibility of Foc. is defined as highly susceptible, susceptible, mildly susceptible, moderate resistant, resistant and highly resistance. Baxijiao, Gongjiao and Guangfen No. 1, are highly susceptible to Foc. TR4. Guangfen No. 1 is highly susceptible to Foc. R1. Haigongiiao and Dajiao are high resistance to Foc. TR4 \& R1. Fenzha No. 1 is high resistance to Foc. TR4 and resistant to Foc. R1, while the infected plants can remain sucker or replanting, the use of biological agents such as Bacillus subtilis also allows plants to restore or ratoon.

\section{Cost Structure and Analysis of Banana Production}

Generally the production and management cost include: land rent, plantation building, plough, buildings and other infrastructure facilities, irrigation system installation, seeds, tools, bagging, support poles (fir or bamboo), fertilizer, pesticides, energy cost, wages, temporary worker's wages, management cadres wages and other incidental expenses etc. Comparative costs are listed in the following Table 2 .

\subsection{Rent, Build Plantation and Other Infrastructure Investment}

Along with economical development, rent of land is also increasing. Moreover the investment also enlarge with the level of production, i.e. infrastructure (work sheds, irrigation and drainage systems, and water source). The spreading of Foc., made many banana plantations become nomadic, moving every $2-3$ years. So the investment of infrastructure is quite big. Land rent of Baxijiao planta- tion is $\$ 1320-2860 / \mathrm{ha} /$ year. Open up wasteland, the preparation of soil in addition, the furrow, the simple water conservation irrigation, $\$ 880-1760 / \mathrm{ha} / \mathrm{crop}$, assessed as two years. Gongjiao's plantation is the same situation. Wasteland is used for Dajiao plantation, the cost is less. Guangfen No. 1's rent per year and a half. The construction costs of $\$ 1057.5 / \mathrm{ha} / \mathrm{crop}$. If former crop is leek for many years, rents of up to $\$ 4400 /$ ha/crop. The cost of rent amounted to $\$ 6600 / \mathrm{ha} / \mathrm{crop}$. The Foc. incidence of Guangfen No. 1 is relatively low in which planted in leek and sugar cane field for years. The harvest rate goes as high as $80 \%-95 \%$. Fenzha No. 1 and Haigongjiao can take advantage of abandoned banana plantation, the rent is cheaper. Fenzha No. 1 takes approximately 13 month to harvest. So the rent is higher. Since these two cultivars can ratoon a few years, other investment-sharing down. The ratoon costs are \$2875.4/ ha/crop and \$ 2391.4/ha/crop respectively. Ratoon costs goes down to $\$ 1760 / \mathrm{ha} / \mathrm{crop}$, and $\$ 1320 / \mathrm{ha} /$ crop respectively.

\subsection{Seedling}

Baxijiao seedling cost $\$ 0.1-0.24 /$ plantlet in different size, approximately cost \$345.4/ha. Dajiao suckers are used which cost \$6.6/ha. Guangfen No. 1 can only use disease-free tissue culture plantlets, $\$ 0.24$ - 0.46/plantlet respectively, approximately cost $\$ 594 /$ ha. Fenzha No. 1 's tissue culture seedling costs $\$ 0.29-0.44$ plantlet, approximately cost $695.2 / \mathrm{ha}$. Gongjiao cost $\$ 0.22 /$ plantlet, approximately cost $\$ 576.4 /$ ha. Haigongjiao cost $\$ 0.22$ 0.26 plantlet in different size respectively, approximately cost \$733.4/ha.

Table 2. The new banana varieties and ratoon production costs (\$/ha).

\begin{tabular}{cccccccccc}
\hline Cultivars & Land rent & Infrastructure & Seeds & Tool bag pole & Feritilizer & Chemical \& Water Labor cost & Other & Total \\
\hline Baxijiao & 2230 & 1103 & 345.4 & 960 & 3275.8 & 585.2 & 1463 & 253 & 10215.5 \\
Baxijiao ratoon & 1650 & 440 & 0 & 581.7 & 3128.8 & 622.4 & 1358.5 & 253 & 8034.4 \\
Dajiao & 1100 & 330 & 290.4 & 111.5 & 671.8 & 76.2 & 733.3 & 66 & 3379.2 \\
Dajiao ratoon & 1100 & 330 & 0 & 111.5 & 671.8 & 76.2 & 733.3 & 66 & 3088.8 \\
Guangfen 1 & 3313.2 & 1057.5 & 590 & 1082.8 & 3379.9 & 906.4 & 1540 & 227.3 & 12097.1 \\
Fenza 1 & 2213 & 663.5 & 695.2 & 1087 & 3439.4 & 507 & 1369.5 & 170.5 & 10149.5 \\
Fenza 1 ratoon & 1324 & 436 & 0 & 435.6 & 2012.4 & 429.6 & 1100 & 148.5 & 5886.1 \\
Gongjiao & 2263 & 1373.6 & 576.4 & 742.5 & 3076.6 & 571 & 1271.6 & 292.6 & 10167.3 \\
Gongjiao ratoon & 1463 & 480.3 & 0 & 586.7 & 1920.5 & 492.2 & 1070.7 & 190.7 & 6204 \\
Haigongjiao & 1719.2 & 673.3 & 733 & 827.9 & 3029.8 & 345 & 1273.4 & 159.5 & 8761.3 \\
Haigongjiao ratoon & 990 & 330 & 0 & 513.3 & 1577.2 & 256.1 & 843.3 & 88 & 4597.8 \\
\hline
\end{tabular}

*Guangfen 1 can not ratoon crop for Foc. race 1 demage. 


\subsection{Tools, Bagging and Banana Pole}

The new tools cost $\$ 22-44 / \mathrm{ha} /$ crop for the Baxijiao plantations with 2 - 3 years. The disposable bunch bags cost $\$ 264$ - 396/ha/crop. The banana pole (fir or bamboo) cost $\$ 0.15-0.74 /$ plant which can be used 1 - 3 years, a total of $\$ 960 / \mathrm{ha} / \mathrm{crop}$, ratoon is $\$ 581.7 / \mathrm{ha} / \mathrm{crop}$. Dajiao's tools cost $\$ 11 / \mathrm{ha} /$ crop. The bunch covers with black film bag that cost $\$ 44-66 /$ ha/crop. \$22/ha/crop for banana pole (in most cases without pole), a total of $\$ 110.9 / \mathrm{ha} /$ crop, and the ratoon are the same. Guangfen No. 1 do not need bagged. It's higher yield in summer and autumn. Pole surport is necessary after flowering. Some plants are dead before harvest, so that a total of $\$ 1082.8 / \mathrm{ha} / \mathrm{crop}$. Fenzha No. 1 does not require bagging, but pole is needed after flowered during summer and autumn because of high yields. In winter, no pole is need because of lower yield, a total cost $\$ 1087.2 / \mathrm{ha} / \mathrm{crop}$, ratoon $\$$ $435.6 / \mathrm{ha} / \mathrm{crop}$. Gongjiao is slender, some time need pole. The bags cost $\$ 198 / \mathrm{ha} / \mathrm{crop}$, a total of $\$ 634.3 / \mathrm{ha} / \mathrm{crop}$, ratoon $\$ 585.2 / \mathrm{ha} /$ crop. Haigongiao's new tool cost $\$ 22$ - 33/ha for 2 - 3 years, bagging \$231/ha/crop, pole \$ 110/ha/crop, a total cost $\$ 827.9 / \mathrm{ha} / \mathrm{crop}$, ratoon $\$$ 513.4/ha/crop.

\subsection{Fertilizer, Pesticides and Water Cost}

The cost of fertilizer for Baxijiao is general $\$ 0.88-\$$ $1.76 /$ plant. Total fertilizer is $\$ 1770-3537 / \mathrm{ha} / \mathrm{crop}$. The cost of irrigation and drainage depend on geography and climate, the energy of pump for irrigation and drain- age costs \$ $0.04-0.29 /$ plant. Pesticide and fungicide costs are $\$ 0.08-0.21$ plant in humid regions. The total cost is $\$ 585.2 /$ ha. Ratoon costs are reduced to $\$ 527 /$ ha. Dajiao usually require less fertilizer, the fertilizer from former crops was used, and the cost is $\$ 0.29-0.37 /$ plant. Pesticide and power cost $\$ 0.044$ plant, $\$ 0.03 /$ plant re- spectively, integrated $\$ 784 /$ ha. The cost of fertilizer for Guangfen No. 1 is $\$ 1.03-2.35$ plant, organic fertilizer, microbial fertilizer cost $\$ 0.88-1.62 /$ plant. Foc. incidenced plants would stop fertilizer. Pesticide and power cost \$ 0.074/plant, \$ 0.12/plant respectively, integrated \$ 4285.6/ha. Fenzha No. 1 cost of fertilizers is $\$ 0.74$ $2.06 /$ plant, pesticides $\$ 0.74$ plant, integrated \$ 3944.6/ha. Ratoon's fertilizer and pesticides are significantly reduced to $\$ 2442 /$ ha. Gongjiao cost of fertilizer $\$ 0.74$ 1.76 plant, mainly organic fertilizer and microbial fertilizer. Pesticide cost \$ 0.074/plant, power cost \$ 0.06/plant, integrated \$ 3647.6/ha. Gongjiao's ratoon cycle is short, only cost $\$ 2411.2 /$ ha. Haigongjiao cost of fertilizer is $\$$ $0.44-1.03$ plant. Pesticide and power cost $\$ 0.74$ plant and $\$ 0.06 /$ plant respectively, integrated $\$ 3385.8 / \mathrm{ha}$, the ratoon cycle is shorter, only $\$ 1832.6 / \mathrm{ha}$.

\subsection{Labor Cost}

Due to the healthy development of economic benefit of the banana industry in recent years; the wages of Industrial workers increase year by year. The labor costs increased accordingly. Labor costs including: the wage of fixed workers, temporary workers and managers. The fixed wage of banana workers costs \$ 132.35 - 191.18/ month, a post culture $1.0-1.33$ ha of land, plus manager's salaries $\$ 132.35 / 1.0-1.33$ ha/year. Converted into a payment linked to output for contract workers: $\$$ $0.0353-0.0441 / \mathrm{kg}$ of banana and manager: $\$ 0.0147 / \mathrm{kg}$ of banana, temporary worker's wage is according to season, region and the different types of work about $\$ 4.41$ 8.82 per day, temporary worker work from planting to harvest (bunch transport) in each post costs about \$ $117.6 /$ crop. Dajiao are mostly planted by locally, labor costs of local farmer is about $\$ 1764.7$ for 2 ha, rarely requested temporary workers. The worker wage for Guagfen No. 1 costs \$ 132.35 - 191.18/month for 1.33 ha. Manager wage is $\$ 132.35 /$ year for $1.3 \mathrm{ha}$, also in the Fenzha No. 1 reference to Baxijiao. The fixed wages of worker for Fenjzha No. 1 are $\$ 132.35$ - 191.18/person/ month for post of $1-1.33$ ha, plus $\$ 132.35 /$ ha/year of manager's salaries. Converted into a payment linked to output for contract worker: $\$ 0.0382-0.0441 / \mathrm{kg}$ of banana and manager $\$ 0.0147 / \mathrm{kg}$ of banana, each post costs about $\$ 44.12$ for temporary worker. Fixed wage of worker for Gongjiao is $\$ 132.35$ - 191.18/person/month for post of $1-1.33$ ha, plus $\$ 132.35 /$ ha/year of manager's salaries. Converted into a payment linked to output for contracted worker: $\$ 0.0588-0.0882 / \mathrm{kg}$ of banana and manager $\$ 0.0294 / \mathrm{kg}$ of banana, each post costs about $\$ 44.12$ for temporary worker. Fixed wage of worker for Haigongjiao is $\$ 132.35$ - 191.18/person/month for post of $1-1.33$ ha, plus $\$ 132.35 /$ ha/year of manager's salaries. Converted into a payment linked to output, contracted worker: $\$ 0.0588-0.0882 / \mathrm{kg}$ of banana and manager $\$ 0.0294 / \mathrm{kg}$ of banana, each post costs about $\$ 44.12$ for temporary worker.

\subsection{Other}

Other costs include: Referral fee for rent land, public relations meals, security fees, transportation, communications, medical expenses, festivals fees. Dajiao and Haigongiiao is the use of local old plantation, other expenses are less. Guangfen No. 1 is planted in plant crop, with longer growing cycle cost higher. Baxijiao, Fenzha No. 1, Gongjiao basically are same.

\subsection{Total Cost}

Baxijiao plant crop costs $\$ 10234.4 /$ ha, ratoon cost is $\$$ 8034.4 /ha that reduce to build plantation, seeds, banana 
pole, fertilizer, labor and other costs. Dajiao plant crop costs \$ 3379.2/ha. Ratoon costs \$ 3088.8/ha, save the seed only. Guanfen No. 1 costs \$12095.6/ha. Fenzha No. 1, plant crop costs $\$ 10144.2 /$ ha. Ratoon cycle is shorter, no plantation build, banana pole, less fertilizer, labor costs, so the cost dropped to $\$ 5885 / \mathrm{ha}$. New Gongjiao costs $\$ 10166.2 / \mathrm{ha}$, ratoon cycle is shorter time, the cost of plantation build, banana pole, fertilizer, labor are lower, so the cost dropped to $\$ 6204 / \mathrm{ha}$. New Haigongjiao costs $\$ 8760.4 /$ ha, ratoon cycle is 3 - 5 months shorter, plantation building, banana pole, fertilizer, labor cost are cut down, the cost dropped to $\$ 4589 /$ ha.

\section{Cost Benefit Analysis}

Farm's surveys of various kinds of banana cultivars, the average purchase price, calculated after deducting costs, output and profits, and conversion out of annual profit as Table 3.

\subsection{Purchase Price}

The purchase price refers to the collection price in farms, including harvesting and transport to the packaging house costs, excluding packing materials and labor costs, in Guangdong province, the purchase price refers to the gross mass of bunch prices, in Hainan, that is the net weight prices. In some cases, Sometimes the price is classified by the yield, the looking and season. This paper calculated according to average price. Cavendish top price is up to $\$ 0.824 / \mathrm{kg}$, the lowest is $\$ 0.12 / \mathrm{kg}$; Dajiao's top price is up to $\$ 0.353 / \mathrm{kg}$, lower price $\$$ $0.074 / \mathrm{kg}$ in July-early September. Guangfen No. 1's top price is up to $\$ 1.029 / \mathrm{kg}$. Gongjiao and Haigongjiao are soled in good prices in Hainan. Gongjiao price is up to \$ $1.853 / \mathrm{kg}$. Haigongjiao's top price is $\$ 1.559 / \mathrm{kg}$. Lower is $\$ 0.441 / \mathrm{kg}$. The Price of Guangfen No. 1 in Pearl River Delta is higher than Haigongjiao, because of the flavor and market demand. If Guangfen No. 1 is soled Northern China, the prices should rise.

\subsection{First Crop Output}

First crop output is the purchase price multiplied the yield. Cavendish Baxijiao is $\$ 14708.8 / \mathrm{ha} / \mathrm{crop}$; Dajiao is
\$ 4622.9/ha/crop; Guangfen No. 1 is \$ 20824.1/ha/crop; Fenzha No. 1 is $\$ 15798.2 /$ ha/crop; Gongjiao is $\$ 18168.3$ /ha/crop; Haigongjiao is $\$ 14502.6 / \mathrm{ha} / \mathrm{crop}$.

\subsection{First Crop Profit}

First crop profit is output value of the plant crop's minus the production costs. Table 3 showed that the plant crop profits are generally not high. Cavendish Baxijiao is $\$$ 4493.3/ha/crop; Dajiao is \$1243.7/ha/crop; Guangfen No. 1 is $\$ 8325.1 / \mathrm{ha} /$ crop; Fenzha No. $1 \$ 5648.7 / \mathrm{ha} / \mathrm{crop}$; Gongjiao is $\$ 8001.0 /$ ha/crop; Haigongiiao is $\$ 5741.3 /$ ha/crop. Guangfen No. 1 and Gongjiao are high risk susceptible to Foc. The investigated plantations were luckily prevented and control, so that the better harvests were obtained.

\subsection{First Crop Annual Profit}

The first crop annual profit is the plant crop profit converted plant crop's cycle to 365-day's value. Cavendish Baxijiao is \$ $5190.0 / \mathrm{ha} / \mathrm{crop}$; Dajiao is \$ $1287.6 / \mathrm{ha} / \mathrm{crop}$; Guangfen No. 1 is $\$ 6684.5 /$ ha/crop; Fenzha No. 1 is $\$$ $5527.5 / \mathrm{ha} /$ crop; Gongjiao is \$ $10319.3 / \mathrm{ha} / \mathrm{crop}$; Haigongjiao is $\$ 9357.0 / \mathrm{ha} / \mathrm{crop}$. Guangfen No. 1 and Fenzha No. 1 the annual profit is not high because of longer cycle. The shorter cycle one Haigongjiao and Gongjiao are better efficiency.

\subsection{Ratoon Output}

The ratoon output is the output multiplied by the price. Cavendish Baxijiao is $\$ 13659.4 / \mathrm{ha} / \mathrm{crop}$; Dajiao is $\$$ 4552.5/ha/crop; Fenzha No. 1 is $\$ 19375.2 /$ ha/crop; Gongjiao is $\$ 16970.4 / \mathrm{ha} / \mathrm{crop}$; Haigongjiao is $\$ 14997.6 / \mathrm{ha} /$ crop. Ratoon banana production generally affected by the harvesting rate, bunch weight and purchase price.

\subsection{Ratoon Profit}

The ratoon profit is output value of the ratoon crop minus the production costs. Due to lower production costs, the ratoon profits generally higher than the plant crop, especially the cultivars that resistant to Foc. It would show advantage after second ratoon except lower harvest rate. Cavendish Baxijiao is $\$ 5625.0 / \mathrm{ha} / \mathrm{crop}$; Dajiao is $\$$

Table 3. The cultivars of the income statement.

\begin{tabular}{|c|c|c|c|c|c|c|c|}
\hline Cultivars & $\begin{array}{l}\text { Collecting price } \\
\qquad(\$ / \mathrm{kg})\end{array}$ & $\begin{array}{l}\text { Crop output } \\
\text { (\$/ha/crop) }\end{array}$ & $\begin{array}{l}\text { Crop profit } \\
\text { (\$/ha/crop) }\end{array}$ & $\begin{array}{l}\text { Annual profit } \\
\text { (\$/ha/aunnual) }\end{array}$ & $\begin{array}{l}\text { Ratoon output } \\
\text { (\$/ha/crop) }\end{array}$ & $\begin{array}{l}\text { Ratoon profi } \\
(\$ / \text { ha/crop) }\end{array}$ & $\begin{array}{l}\text { Ratoon annual profit } \\
\text { (\$/ha/aunnual) }\end{array}$ \\
\hline Baxijiao & 0.37 & 14708.8 & 4493.3 & 5190.0 & 13659.4 & 5625.0 & 8736.6 \\
\hline Guangfen No. 1 & 0.67 & 20824.1 & 8325.1 & 6684.5 & - & - & - \\
\hline Fengzha No. 1 & 0.64 & 15798.2 & 5648.7 & 5527.5 & 19375.2 & 1348.9 & 22078.5 \\
\hline Gongjiao & 0.74 & 18168.3 & 8001.0 & 10319.3 & 16970.4 & 10766.4 & 20361.2 \\
\hline Haigongjiao & 0.65 & 14502.6 & 5741.3 & 9357.0 & 14997.6 & 10399.8 & 27113.9 \\
\hline
\end{tabular}


1463.7/ha/crop; Fenzha No. 1 is $\$ 1348.9 / \mathrm{ha} /$ crop; Gongjiao is $\$ 10766.4 / \mathrm{ha} /$ crop; Haigongjiao is $\$ 10399.8 / \mathrm{ha} /$ crop.

\subsection{Ratoon Annual Profit}

Ratoon annual profit is the ratoon crop profit converted ratoon cycle to 365 days values. The Table 3 showed that Fenzha No. 1, Gongjiao and Haigongjiao's profit are obviously higher because of short cycle. This means the land is more productively than Cavendish Baxijiao. Cavendish Baxijiao is $\$ 8736.6 / \mathrm{ha} /$ crop; Dajiao is $\$$ 1942.6/ha/crop; Fenzha No. 1 is \$ 22078.5/ha/crop; Gongjiao is $\$ 20361.2 /$ ha/crop; Haigongjiao is $\$ 27113.9 / \mathrm{ha} /$ crop.

\section{The Existing Problems in the Banana Cultivars}

\subsection{Cavendish (AAA)}

Cultivation risk of Cavendish is higher since the available Foc. TR4 resistant cultivars, named Kangku No. 5, Yueyoukang No. 1 and Nongke No. 1 are poor in economic characters, such as longer growth cycle (12 - 18 months), poor bunch shape less hand number, abnormal fingers in first hand, choking, ratoon rihzome tends to climb out to earth, and the poor cold tolerance [6]. There is no better prevention and treatment of chemical and biological agents to against blight, integrated control technology research and development are slow; highquality banana production technologies is only applied in part of the banana plantations in Hainan province. Technology promotion work needs to be strengthened; Sigatoka become top disease in some production zone. Banana harvested in summer easy becomes green-wateryrot phenomenon before ripening. Cold chain storage and transport large cargo are damaged. This is the reason of low price during summer time. No crop rotation was studied for sustainable production in Cavendish plantation.

\subsection{Dajiao (ABB)}

Cultivation risk of Dajiao is lower.

Advantages: Extensive management, low cost, resistant to Foc. TR4 and fungi. Shortages: low level of commodity production, fruit taste sour during summer and autumn. Panyu Dajiao tissue culture would easy degradation, lower hands dysplasia so that were rejected to sell. The purchase price is generally lower than $\$ 0.10 / \mathrm{kg}$, there is no economic profit; not soled in the northern market.

\subsection{Guangfen No. 1 (ABB)}

Cultivation risk of Guangfen No. 1 is higher.
Advantages: higher purchase price and bunch weight. Shortages: many off types in tissue culture plantlets, there is no effective integrated control techniques against Foc. No resistant varieties and biological agents, the harvest rate is low; economic instability. Fruit packaging, transport technology are to be developed, northern market still to be developed.

\subsection{Fenzha No. 1 (ABB)}

Cultivation risk of Fenzha No. 1 cultivation risk is lower.

Advantages: higher purchase price, resistant to Foc. TR4, virus and fungi. Shortages: off type, twinned and abnormal fingers, lower yield and local consumption. Packaging transport technology and northern China market are to be developed.

\subsection{Gongjiao (AA)}

Cultivation risk of Gongjiao is higher.

Advantages: Top price, shorter life cycle time. Shortages: Low-yielding, not resistant to cold and wind, susceptible to Foc. TR4, CMV, BBTV, integrated control Foc. TR4 technology is not developed yet.

\subsection{Haigongjiao (AA)}

Cultivation risk of Haigongjiao is lower.

Advantages: higher purchase price, resistant to Foc. TR4 and the shortest life cycle. Shortages: low-yield, autumn and winter fruit cracking, dropping after ripened; the flavor is slightly acidic, susceptible to CMV and BBTV, chilling damage. Packaging, storage, transport technology are not yet developed.

\section{Perspective Plan and Suggestions to Enhance the Economic Benefit of Banana Diversity in Foc. TR4 Zones}

\subsection{Cavendish (AAA)}

In order to maintain the annual harvest and good price, Cavendish planting and suckering time should be adjusted to keep the harvest season mainly in winter to the early next summer, supplemented by mid-fall, a small amount of the supply in summer. Fruit is off-season during January to June every year. With higher market demand, the price is stability and good, so the incomes are guaranteed. But June-September sometimes got higher prices because of chilling and typhoon. The bunch weight is the highest, so the revenue is good, but risk for typhoon. Breeding to disease resistance should be enhanced in Cavendish as well as integrated control technology and high-quality banana production technology should be improved. The resistant cultivars: Kangku No. 5, Nongke No. 1 etc. are planted in Foc. TR4 areas and the benefits are more secure in Foc. TR4 areas. The cul- 
tivation techniques R \& D (cold resistance, bunch protection, fertilizer, irrigation, off-type, cropping systems etc.) should be highlighted to screen the new clones to improve disease resistance and early flowering. Research should be developed on purification for seedlings, the storage and transportation technology for rotten fruits in summer, as well as rotation crops. In the future the input of Cavendish plantations was as low as about $\$ 6600$ $11000 /$ ha and the profits access to $\$ 4400-11000 /$ ha.

\subsection{Dajiao (ABB)}

The promotion of the productive Dongguan Dajiao, as well as Fendajiao (ABB) and other sweet plantains are necessary. Highlight R \& D for Dajiao bagging, fruit thinning, fertilization and cropping systems. Developing high-quality plantain production order studies, cropping adjustment for harvest in good price, promote it as an organic healthy food in northern market and Dajiao processing to adding value. Evaluating the good taste (sweet, meat hard) Dajiao (AAB, ABB, BBB) these cultivars play a cold, disease resistance, planting low-cost advantages, can be grown in the northern part of South China. So Dajiao can output $\$ 4400 /$ ha, then got $\$ 2200 /$ ha profit, become a farmer an economic cultivation of cash crops.

\subsection{Guangfen No. 1 (ABB)}

The off-type tissue culture plants of Guangfen No. 1 should be eliminated during the greenhouse and 15 leaves plants. Guangfen No. 1 can be extended to virgin land of central district in Guangdong, Guangxi, Fujian provinces. In the case flowering in cold, bagging as the bug emergence, spraying cold-resistant medicament to reduce the formation of young fruit that were frostbitten fruit deformity dysplasia. Enhance the Foc. Integrated control techniques, such as from the plantation selections, the fertilizer, water management, disease control, packaging, leek rotation, cold chain logistics technology research and development, protocol development promo tion. As a high-quality, healthy food, Guangfen No. 1 should step up marketing in the northern China. So Guangfen No. 1 can promote sustainable development, invests \$ 6600 - 13200/ha, incomes \$ 4400 - 11000/ha of profits.

\subsection{Fenzha No. 1 (ABB)}

The off-type tissue culture plants of Fenzha No. 1 were eliminated in the greenhouse and large leaves 15 plants. Thinning fingers to minimize abnormal fingers when cut bud. In the case flowering in cold, bagging as the bug emergence, spraying cold-resistant medicament to reduce the formation of young finger that were frostbitten fruit deformity dysplasia. Highlight R \& D cultivation, packaging, cold chain logistics technologies so that increasing revenue. As a health food, Fenzha No. 1 should step up marketing in the north to ensure sustainable production. Suppose invest \$ 5500 - 9900/ha incomes \$ 4400 $11000 /$ ha profit, and can cropping $3-4$ years. Yatsen Fenjiao, the high yield fenjiao was screened from Fanzha No. 1 by writers in Zhongshan city, Guangdong province in 2009 , which is more than $30 \mathrm{~kg}$ bunch mass, good bunch shape, taste good and resistant to Fusarium wilt race $1 \& 4$, Sigatoka, Fleckle and virus. The life cycle is 1 month longer than Fanzha No. 1. It will be released in 2010. Rosamonde Fenjiao was screened from Fanzha No. 1 by writers in Ledong County, Hainan province in 2009, the Max bunch weight was $41 \mathrm{~kg}$.

\subsection{Gongjiao (AA)}

Strengthen R \& D for Foc. TR4 integrated control, quality cultivation, bunch protection, packaging, coldchain logistics, and the cultivation of technical schedule. As Gongjiao is the best quality, high value banana, there is big potential market can be planted in the new zone. Suppose input \$ 6160 - 10120/ha, incomes \$ 6600 $13200 /$ ha of profits.

\subsection{Haigongjiao (AA)}

Strengthen R \& D to obtain low or no fruit cracking, taste slightly acidic, low-yield etc. Applies fertilizer increases sweetness. Formulates the high quality Haigongjiao production order and promotes in the plantations. The packing, the cold chain logistics technology should be worked out. Promote the product in the north market. Because of short life cycle, it can be planted in northern territory. Suppose input \$ $4400-8800 / \mathrm{ha}$, incomes \$ 4400 - 11000/ha profits.

\subsection{Rotation}

Rotation crop celection should adapte according to local conditions. The first crop for the virgin land could be Cavendish, Gongjiao and Fenjiao for 1 - 3 years. In case the plantation was infected by Foc. TR4, then it rotate to Fenzha No. 1, Haigongjiao and disease-resistant Cavendish, and then rotate to other crops after 3 - 5 years. And sweet corn, papaya, vegetables, sugar cane can be planted since they do not need to carry on the furrow, the preparation of soil, the deep plowing, the fixed facility and special irrigation system. According to time limit of the land, other crops rotate for 3 - 5 years, and then bananas can be planted again. So it can save the cost on labor, trace element fertilizers, chemical, soil cultivation, and reducing diseases and pest, becoming a virtuous circle. According to the author investigates the leek rotation and the inter-species may delay plant disease to occur, and decrease the Foc. TR4 incidence rate. The responsive principle waits for further study to achieve the goal of 
sustainable production of banana.

\section{Acknowledgements}

These studies were sponsored by the project of the key technologies R \& D for banana industry promotion and Guangdong Modern agricultural technology system: Lingnan fruit industry innovation and team building system.

\section{REFERENCES}

[1] A. B. Molina, "Banana Fusarium Wilt Management: Towards Sustainable Cultivation," The International Network for the Improvement of Banana and Plantain, Monterpeller, 1999, pp. 7-8.

[2] FAOSTAT, 2011.

http://faostat.fao.org/site/567/default.aspx\#an-cor
[3] Peikun Qi, "Status Report of Banana Fusarium Wilt Disease in China, Banana Fusarium Wilt Management: Towards Sustainable Cultivation, The International Network for the Improvement of Banana and Plantain, Monterpeller, 1999, pp. 118-120.

[4] Bingzhi Huang, Linbing Xu, et al., "Preliminary Evalution of IMTP-III Varities and Local Cultivars against Fusarium Wilt Disease in South China, Advancing Banana and Plantain R \& D in Asia and the Pacific-Vol. 13," The International Network for the Improvement of Banana and Plantain, Monterpeller, 2004, 187-191.

[5] Linbing Xu, et al., "Practical Technology for Banana Production," Guangdong Science and Technology Press, Guangzhou, 2008, pp. 13-16.

[6] Linbing Xu, et al., "The Atlas of Banana Cultivars and Cultivation Technology," China Agriculture Press, Beijing, 2008, pp. 35-45. 